Annals of Pure and Applied Mathematics

Vol. 12, No. 2, 2016, 221-232

ISSN: 2279-087X (P), 2279-0888(online)

Published on 14 December 2016

www.researchmathsci.org

DOI: http://dx.doi.org/10.22457/apam.v12n2a13

Annals of

Pure and Applied

Mathematics

\title{
Global Theory of Smooth Functions of Manifolds
}

\author{
Mohammad Raquibul Hossain ${ }^{1}$, Md. Raknuzzaman ${ }^{2}$ \\ Md. Mijanoor Rahman ${ }^{3}$ and Md. Jamal Hossain ${ }^{4}$ \\ ${ }^{1,4}$ Department of Applied Mathematics, Noakhali Science \& Technology University \\ Noakhali-3814, Bangladesh. E-mail: raquib.math@gmail.com; z math_du@yahoo.com \\ ${ }^{2}$ Department of Arts and Sciences, Ahsanullah University of Science and Technology \\ Dhaka, Bangladesh. Email: rokon011@gmail.com \\ ${ }^{3}$ Department of Mathematics, Mawlana Bhasani Science \& Technology University \\ Santos, Tangail-1902, Bangladesh. Email: mizanmath@yahoo.com \\ ${ }^{1}$ Corresponding author
}

Received 4 November 2016; accepted 30 November 2016

Abstract. Our present goal is to extend the theory of smooth functions, developed on open subsets of $\mathbb{R}^{\mathrm{n}}$ in the global theory of smooth functions to arbitrary differentiable manifolds, in this case geometric topology becomes an essential feature.

Keywords: Topology, differential geometry, algebraic topology, manifold

AMS Mathematics Subject Classification (2010): 57R19, 58A05

\section{Introduction}

Fundamental to the global theory of differentiable manifolds is the concept of a vector bundle. The easiest nontrivial example is the tangent bundle to the $n$-sphere which we introduce from a purely topological point. The subtleties involved in this bundle are illustrated in the discussion of the vector field problem. As the global theory is developed, the tangent bundle, the cotangent bundle, various tensor bundles, and the associated (principal) frame bundles will play increasingly important roles [3], as will the related notions of infinitesimal $\mathrm{G}$-structures and integrable $G$-structures. For conceptual simplicity, all manifolds, functions, bundles, vector fields, Lie groups, homogeneous spaces, etc., will be smooth of class $C^{\infty}$. We study Smooth manifolds and mapping, diffeomorphic structures, the tangent bundle, cocycles and geometric structures, global construction of smooth functions, manifolds with boundary and finally submanifolds.

\section{Smooth manifolds and mapping}

Let $M$ be a topological manifold of dimension $n$. The locally Euclidean property allows us to choose local coordinates in any small region of $M$.

Definition 2.1. A coordinate chart [2] on $M$ is a pair $(U, \varphi)$, where $U \subseteq M$ is an open subset and $\varphi: U \rightarrow \mathbb{R}^{n}$ is a homeomorphism onto an open subset $\mathbb{R}^{n}$.

We usually write $\varphi(p)=\left(x^{1}(p), \cdots \cdots, x^{n}(p)\right)$, viewing this as the coordinate $n$-tuple of the point $p \in U$. 
M.R.Hossain, Md. Raknuzzaman, Md. Mijanoor Rahman and Md. Jamal Hossain

Definition 2.2. Two coordinate charts $(U, \varphi)$ and $(V, \psi)$ on $M$ are said to be $C^{\infty}$ related if either $U \cap V=\emptyset$ or

is diffeomorphism.

$$
\varphi \circ \psi^{-1}: \psi(U \cap V) \rightarrow \varphi(U \cap V)
$$

Definition 2.3. A $C^{\infty}$ atlas on $M$ is a collection $\mathcal{A}=\left\{\left(U_{\alpha}, \varphi_{\alpha}\right)\right\}_{\alpha \in \mathfrak{A}}$ of coordinate charts such that

(i) $\left(U_{\alpha}, \varphi_{\alpha}\right)$ is $C^{\infty}$ related to $\left(U_{\beta}, \varphi_{\beta}\right), \forall \alpha, \beta \in \mathfrak{U}$

(ii) $M=\cup_{\alpha \in \mathfrak{A}} U_{\alpha}$

Two $C^{\infty}$ atlas $\mathcal{A}$ and $\mathcal{A}^{\prime}$ on $M$ are equivalent if $\mathcal{A} \cup \mathcal{A}^{\prime}$ is also a $C^{\infty}$ atlas on $M$.

Example 2.4. The manifold $\mathbb{R}^{n}$ has a canonical smooth structure [1], namely the set $\mathcal{A}_{n}$ of all pairs $(U, \varphi)$ where $U \subseteq \mathbb{R}^{n}$ is open and $\varphi: U \rightarrow \mathbb{R}^{n}$ is a diffeomorphism onto an open set $\varphi(U) \subseteq \mathbb{R}^{n}$.

Example 2.5. If $M$ is a smooth $m$-manifold and $N$ a smooth $n$-manifold with respective smooth structures $\mathcal{A}=\left\{\left(U_{\alpha}, \varphi_{\alpha}\right)\right\}_{\alpha \in \mathfrak{A}}$ and $\mathcal{B}=\left\{\left(V_{\beta}, \psi_{\beta}\right)\right\}_{\beta \in \mathfrak{B}}$, then $M \times N$ is canonically a smooth $(m+n)$ manifold. Indeed

$$
\mathcal{A} \times \mathcal{B}=\left\{\left(U_{\alpha} \times V_{\beta}, \varphi_{\alpha} \times \psi_{\beta}\right)\right\}_{(\alpha, \beta) \in \mathfrak{A} \times \mathfrak{B}}
$$

is a $C^{\infty}$ atlas, determining uniquely a maximal one, called the Cartesian product of the two smooth structures.

Definition 2.6. Let $M$ be a smooth $n$-manifold with smooth atlas $\mathcal{A}=\left\{\left(U_{\alpha}, \varphi_{\alpha}\right)\right\}_{\alpha \in \mathfrak{A} \text {. }}$ Set the function

$$
g_{\alpha \beta}=U_{\alpha} \circ \varphi_{\beta}^{-1}: \varphi_{\beta}\left(U_{\alpha} \cap U_{\beta}\right) \rightarrow \varphi_{\alpha}\left(U_{\alpha} \cap U_{\beta}\right)
$$

These local diffeomorphisms in $\mathbb{R}^{n}$ satisfying the cocycle conditions

$$
\begin{aligned}
& \text { (i) } g_{\alpha \beta} \circ g_{\beta \gamma}=g_{\alpha \gamma} \\
& \text { (ii) } g_{\alpha \alpha}=i d_{\varphi_{\alpha}\left(U_{\alpha}\right)} \\
& \text { (iii) } g_{\beta \alpha}=g_{\alpha \beta}^{-1}
\end{aligned}
$$

It should be noted that the properties (ii) and (iii) follows from the property $(i)$.

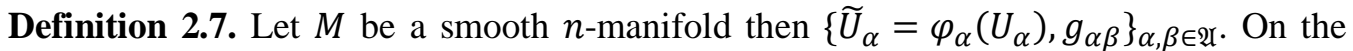
disjoint union

define the relation

$$
\widetilde{M}=\bigsqcup_{\alpha \in \mathfrak{A}} \widetilde{U}_{\alpha}
$$

$$
x \sim y \Leftrightarrow \exists \alpha, \beta \in \mathfrak{A} \text { such that } x \in \widetilde{U}_{\alpha}, y \in \widetilde{U}_{\beta} \text { and } y=g_{\beta \alpha}(x) .
$$

By properties (i), (ii) and (iii) of the definition 1.06, this is an equivalence relation, so we form the topological quotient space $\tilde{M} / \sim$. We will show that this space is homeomorphic to $M$ and exhibit a natural smooth structure on it.

Let $[z] \in \widetilde{M} / \sim$ denote the equivalence class $z \in \widetilde{M}$. Define

$$
\varphi: M \rightarrow \tilde{M} / \sim
$$

By setting $\varphi(x)=\left[\varphi_{\alpha}(x)\right]$ if $x \in U_{\alpha}$. If $x \in U_{\beta}$ also then $g_{\alpha \beta}\left(\varphi_{\beta}(x)\right)=\varphi_{\alpha}(x)$, so $\varphi$ is well defined. It is continuous. The map from $\widetilde{M}$ to $M$ that takes $z \in \widetilde{U}_{\alpha}$ to $\varphi_{\alpha}^{-1}(z)$ respects to equivalence relation, hence passes to a continuous map 
Global Theory of Smooth Functions of Manifolds

$$
\varphi: \widetilde{M} / \sim \rightarrow M \text {. }
$$

It is easy to see that $\varphi$ and $\psi$ are mutually inverse, so $M$ and $\widetilde{M} / \sim$ are canonically homeomorphic. Each $\widetilde{U}_{\alpha}$ imbeds canonically in $\widetilde{M} / \sim$ as an open subset and $i d_{\alpha}: \widetilde{U}_{\alpha} \rightarrow$ $\widetilde{U}_{\alpha} \subseteq \mathbb{R}^{n}$ defines a coordinate chart $\left(\widetilde{U}_{\alpha}, i d_{\alpha}\right)$ on $\widetilde{M} / \sim$. These charts are $C^{\infty}$-related via the cocycle $\left\{g_{\alpha \beta}\right\}_{\alpha, \beta \in \mathfrak{H}}$, so $\widetilde{M} / \sim$ is canonically identified with $M$ as a smooth manifold via the mutually diffeomorphisms $\varphi$ and $\psi$.

Definition 2.8. A function $f: M \rightarrow \mathbb{R}$ is said to be smooth if, for each $x \in M$, there exist a chart $(U, \varphi) \in \mathcal{A}$ such that $x \in U$ and

$$
f \circ \varphi^{-1}: \varphi(U) \rightarrow \mathbb{R}
$$

is smooth. The set of all smooth, real valued functions on $M$ will be denoted $C^{\infty}(M)$.

Lemma 2.9. The function $f: M \rightarrow \mathbb{R}$ is smooth if and only if

is smooth, $\forall\left(U_{\alpha}, \varphi_{\alpha}\right) \in \mathcal{A}$.

$$
f \circ \varphi_{\alpha}^{-1}: \varphi_{\alpha}\left(U_{\alpha}\right) \rightarrow \mathbb{R}
$$

Proof: Condition implies that $f$ is smooth. For the converse, suppose that $f$ is smooth and let $x \in U_{\alpha}$ where $\left(U_{\alpha}, \varphi_{\alpha}\right) \in \mathcal{A}$. By definition 1.08 , choose $\left(U_{\beta}, \varphi_{\beta}\right) \in \mathcal{B}$ such that $x \in U_{\beta}$ and

is smooth. Then

$$
f \circ \varphi_{\beta}^{-1}: \varphi_{\beta}\left(U_{\beta}\right) \rightarrow \mathbb{R}
$$

is given by the decomposition

$$
f \circ \varphi_{\alpha}^{-1}: \varphi_{\alpha}\left(U_{\alpha} \cap U_{\beta}\right) \rightarrow \mathbb{R}
$$

$$
\varphi_{\alpha}\left(U_{\alpha} \cap U_{\beta}\right) \stackrel{g_{\beta \alpha}}{\longrightarrow} \varphi_{\beta}\left(U_{\alpha} \cap U_{\beta}\right) \stackrel{f \circ \varphi_{\beta}^{-1}}{\longrightarrow} \mathbb{R}
$$

as a composition of smooth maps, this is smooth. That is,

$$
f \circ \varphi_{\alpha}^{-1}: \varphi_{\alpha}\left(U_{\alpha}\right) \rightarrow \mathbb{R}
$$

is smooth on some neighborhood of the point $\varphi_{\alpha}(x)$. But $x \in U_{\alpha}$ is arbitrary, so $f \circ \varphi_{\alpha}^{-1}$ is smooth on all of $\varphi_{\alpha}\left(U_{\alpha}\right)$.

Definition 2.10. Let $M$ and $N$ be $C^{\infty}$ manifolds with respective smooth structures $\mathcal{A}$ and $\mathcal{B}$. A mapping $f: M \rightarrow N$ is said to be smooth if, for each $x \in M$, there are $\left(U_{\alpha}, \varphi_{\alpha}\right) \in \mathcal{A}$ and $\left(U_{\beta}, \varphi_{\beta}\right) \in \mathcal{B}$ such that $x \in U_{\alpha}, f\left(U_{\alpha}\right) \subseteq V_{\beta}$, and

is smooth.

$$
\psi_{\beta} \circ f \circ \varphi_{\alpha}^{-1}: \varphi_{\alpha}\left(U_{\alpha}\right) \rightarrow \psi_{\beta}\left(V_{\beta}\right)
$$

Definition 2.11. A derivative of $\mathfrak{G}_{p}$ is a $\mathbb{R}$ - linear map

such that

$$
D: \mathfrak{b}_{p} \rightarrow \mathbb{R}
$$

$$
D(\xi \zeta)=D(\xi) e_{p}(\zeta)+e_{p}(\xi) D(\zeta)
$$

$\forall \xi, \zeta \in \mathfrak{G}_{p}$. This operator $D$ is called a tangent vector to $M$ at $p$ and the vector space $T_{p}(M)$ of all derivative of $\mathfrak{G}_{p}$ is called the tangent space [11] to $M$ at $p$.

Definition 2.12. If $f: M \rightarrow N$ is a smooth map between manifolds and if $p \in M$, the differential 
M.R.Hossain, Md. Raknuzzaman, Md. Mijanoor Rahman and Md. Jamal Hossain is the linear map defined by

$$
f_{* p}=d f_{p}: T_{p}(M) \rightarrow T_{f(p)} M
$$

$$
\left(f_{* p}(D)\right)[g]_{f(p)}=D[g \circ f]_{p},
$$

for all $D \in T_{p}(M)$ and all $[g]_{f(p)} \in \mathfrak{G}_{f(p)}$.

Lemma 2.13. If $f: M \rightarrow N$ and If $g: N \rightarrow P$ are smooth map between manifolds and $x \in M$, then $d(g \circ f)_{x}=d g_{x} \circ d f_{x}$.

Proof: Consider

$$
\begin{aligned}
\left((g \circ f)_{* p}(D)\right) & {[h]_{g(f(p))}=D[h \circ f \circ g]_{p} } \\
& =\left(f_{* p}(D)[h \circ g]_{f(p)}\right) \\
& =\left(g_{* f(p)}\left(f_{* p}(D)\right)\right)[h]_{g(f(p))}
\end{aligned}
$$

Since $[h]_{g(f(p))} \in \mathfrak{G}_{g(f(p))}$ and $D \in T_{p}(M)$ are arbitrary.

Corollary 2.14. If $M$ is a smooth manifold of dimension $n$, then $T_{x}(M)$ is a real vector space of dimension $n, \forall p \in M$.

Proof: Let $(U, \varphi)$ be a coordinate patch on $M$ with $x \in U$. Then $T_{x}(U)=T_{x}(M)$ so we have

$$
\varphi_{* x}: T_{x}(U) \rightarrow T_{\varphi(x)}(\varphi(U))
$$

is an $\mathbb{R}$ - linear isomorphism. Since $\varphi(U) \in \mathbb{R}^{n}$ is open, we know that

$$
T_{\varphi(x)}(\varphi(U))=\mathbb{R}^{n}
$$

\section{Diffeomorphic structures}

Diffeomorphism is an isomorphism in the category of smooth manifolds. It is an invertible function that maps one differentiable manifold to another, such that both the function and its inverse are smooth functions.

This section is really an extended remark on some very deep theorems. For this purpose, let $M$ be a differentiable manifold with smooth structure [10] $\mathcal{A}=$

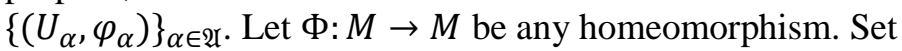

$$
\mathcal{A}_{\Phi}=\left\{\left(\Phi^{-1}\left(U_{\alpha}\right), \varphi_{\alpha} \circ \Phi\right)\right\}_{\alpha \in \mathfrak{A}} .
$$

Proposition 3.1. The set $\mathcal{A}_{\Phi}$ is a $C^{\infty}$ structure on $M$ having the same structure cocycle as $\mathcal{A}$.

Proof: We know

and this map carries the set

$$
g_{\alpha \beta}=\varphi_{\alpha} \circ \varphi_{\beta}^{-1}=\left(\varphi_{\alpha} \circ \Phi\right) \circ\left(\varphi_{\beta} \circ \Phi\right)^{-1},
$$

$$
\left(\varphi_{\beta} \circ \Phi\right)\left(\Phi^{-1}\left(U_{\alpha}\right) \cap \Phi^{-1}\left(U_{\beta}\right)\right)=\varphi_{\beta}\left(U_{\alpha} \cap U_{\beta}\right)
$$

onto the set

$$
\left(\varphi_{\alpha} \circ \Phi\right)\left(\Phi^{-1}\left(U_{\alpha}\right) \cap \Phi^{-1}\left(U_{\beta}\right)\right)=\varphi_{\alpha}\left(U_{\alpha} \cap U_{\beta}\right)
$$

Finally, the maximality of the $C^{\infty}$ atlas $\mathcal{A}_{\Phi}$ follows from that of $\mathcal{A}$ and the fact that $\mathcal{A}_{\Phi \Phi^{-1}}=\mathcal{A}$.

Definition 3.2. Two $C^{\infty}$ structures [6] $\mathcal{A}$ and $\mathcal{B}$, defined on some topological manifold $M$, are said to be diffeomorphic structures $\mathcal{B}=\mathcal{A}_{\Phi}$, for some homeomorphism $\Phi: M \rightarrow M$. 
Global Theory of Smooth Functions of Manifolds

Example 3.3. Let $\sigma(n)$ denote the number of diffeomorphism classes of differentiable structures on $S^{n}$. It was long known that $\sigma(n)=1$ for $n=1,2,3$. The value of $\sigma(4)$ remains mystery. The following table for $5 \leq n \leq 18$ was computed by Kervaire and Milnor.

\begin{tabular}{|c|l|l|l|l|l|l|l|l|l|l|c|c|c|c|}
\hline$n$ & 5 & 6 & 7 & 8 & 9 & 10 & 11 & 12 & 13 & 14 & 15 & 16 & 17 & 18 \\
\hline$\sigma(n)$ & 1 & 1 & 28 & 2 & 8 & 6 & 992 & 1 & 3 & 2 & 16256 & 2 & 16 & 16 \\
\hline
\end{tabular}

\section{The tangent bundle}

Let $M$ be a $C^{\infty} n$-maifold with structure $\left\{\left(U_{\alpha}, \varphi_{\alpha}\right)\right\}_{\alpha \in \mathfrak{A} \text {. Consider the set }}$

$$
T=\bigsqcup_{x \in M} T_{x}(M)
$$

a disjoint union with, as set no topological structure. For each $U_{\alpha}, \alpha \in \mathfrak{A}$, define

$$
T\left(U_{\alpha}\right)=\bigsqcup_{x \in U_{\alpha}} T_{x}(M) \subseteq T .
$$

Then the individual linear maps $d \varphi_{\alpha x}, x \in U_{\alpha}$, unite a define a set map

$$
d \varphi_{\alpha}: T\left(U_{\alpha}\right) \rightarrow T\left(\varphi_{\alpha}\left(U_{\alpha}\right)\right)=\varphi_{\alpha}\left(U_{\alpha}\right) \times \mathbb{R}^{n} \subseteq \mathbb{R}^{2 n}
$$

More precisely, if $v_{x}$ denotes a tangent vector to $M$ at $x \in U_{\alpha}$

$$
d \varphi_{\alpha}\left(v_{x}\right)=\left(\varphi_{\alpha}(x), d \varphi_{\alpha x}\left(v_{x}\right)\right)
$$

and this defines a bijection of $T\left(U_{\alpha}\right)$ onto an open subset of $\mathbb{R}^{2 n}$. If $U_{\alpha} \cap U_{\beta} \neq \emptyset$, consider

By the chain rule

$$
d \varphi_{\alpha} \circ d \varphi_{\beta}^{-1}: T\left(\varphi_{\beta}\left(U_{\alpha} \cap U_{\beta}\right)\right) \rightarrow T\left(\varphi_{\alpha}\left(U_{\alpha} \cap U_{\beta}\right)\right)
$$

a $C^{\infty}$ diffeomorphism between the open subsets of $\mathbb{R}^{2 n}$.

We topologize the set $T$. If

$$
W \subseteq d \varphi_{\alpha}\left(T\left(U_{\alpha}\right)\right)=T\left(\varphi_{\alpha}\left(U_{\alpha}\right)\right.
$$

is an open set, then $d \varphi_{\alpha}^{-1}(W)$ is to be an open subset of $T$.

Definition 4.1. The system $\pi: T M \rightarrow M$ is said called the tangent bundle [5] of $M$. The total space $T(M)$, the base space is $M$, and $\pi$ is called the bundle projection.

Definition 4.2. A vector field on $M$ is a smooth map $X: M \rightarrow T(M)\left(p \mapsto X_{p}\right)$ such that $\pi \circ X=i d_{M}$. The set of all vector fields on $M$ is denoted by $\mathfrak{X}(M)$.

Remark: Let $X$ be a vector field on $M,\left(U, x^{1}, \cdots \cdots, x^{n}\right)$ a coordinate chart on $M$. Then

where $f^{i}: U \rightarrow \mathbb{R}$ is smooth, $1 \leq i \leq n$.

$$
X \mid U=\sum_{i=1}^{n} \frac{\partial}{\partial x^{i}}
$$

Definition 4.3. Let $M$ be a smooth $m$-manifold, $E$ a smooth $(m+n)$ - manifold, and $\pi: E \rightarrow M$ a smooth map. This will be called an $n$-plane bundle over $M$ (or a vector bundle over $M$ of fiber dimension $n$ ) if the following properties hold

(i) For each $x \in M, E_{x}=\pi^{-1}(x)$ has the structure of real, $n$-dimensional vector space.

(ii) There is an open cover $\left\{W_{j}\right\}_{j \in J}$ of $M$, together with commutative diagrams

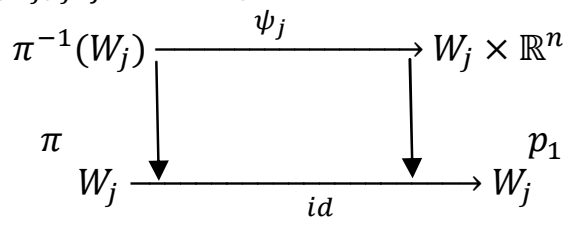


M.R.Hossain, Md. Raknuzzaman, Md. Mijanoor Rahman and Md. Jamal Hossain such that $\psi_{j}$ is diffeomorphism, $\forall j \in J$.

(iii) For each $j \in J$ and $x \in W_{j}, \psi_{j x}=\psi_{j} \mid E_{x}$ maps the vector space $E_{x}$ isomorphically onto the vector space $\{x\} \times \mathbb{R}^{n}$.

As with tangent bundles, we call $E$ is the total space, $M$ is the base space and $\pi$ the bundle projection. We also call each $W_{j}$ a trivializing a neighborhood for the bundle and $\left\{W_{j}\right\}_{j \in J}$ a locally trivializing cover (of $M$ ) for $E$.

Definition 4.4. An $n$-plane bundle is trivial if it is a isomorphic to the product bundle $p_{1}: M \times \mathbb{R}^{n} \rightarrow M$.

Definition 4.5. A section of the $n$-tuple is trivial $\pi: E \rightarrow M$ is a smooth map $s: M \rightarrow E$ such that $\pi \circ s=i d_{M}$. The set of all such sections is denoted by $\Gamma(E)$.

Definition 4.6. The manifold $M$ parallelizable if there are fields

$$
X_{1}, X_{2}, X_{3}, \cdots \cdots, X_{n} \in \mathfrak{X}(M)
$$

such that $\left\{X_{1 x}, X_{2 x}, \cdots \cdots, X_{n x}\right\}$ is a basis of $T_{x}(M), \forall x \in M$.

Example 4.7. We give 1-plane bundle (a line bundle) over a circle, known as the Möbius bundle. On $\mathbb{R} \times \mathbb{R}$, define the equivalence relation $(s, t) \sim\left(s+n,(-1)^{n} t\right), n \in \mathbb{Z}$. Remark that $t \rightarrow(-1)^{n} t$ is a linear automorphism of $\mathbb{R}$. The projection $(s, t) \rightarrow s$ passes to a well defined map $:(\mathbb{R} \times \mathbb{R}) /(\sim) \rightarrow \mathbb{R} / \mathbb{Z}=S^{1}$. It should be clear, intuitively that this a vector bundle over $S^{1}$ of fiber dimension one.

\section{Cocycles and geometric structures}

Let $\pi: E \rightarrow M$ be an $n$-plane bundle and let $\left\{W_{j}\right\}_{j \in J}$ be a locally trivializing open cover of $E$, the trivializations being $\psi_{j}: \pi^{-1}\left(W_{j}\right) \rightarrow W_{j} \times \mathbb{R}^{n}$. If $W_{i} \cap W_{j} \neq \varnothing$, consider

$$
\left(W_{i} \cap W_{j}\right) \times \mathbb{R}^{n} \stackrel{\psi_{i}^{-1}}{\longrightarrow} \pi^{-1}\left(W_{i} \cap W_{j}\right) \stackrel{\psi_{j}}{\longrightarrow} \pi^{-1}\left(W_{i} \cap W_{j}\right) \times \mathbb{R}^{n} .
$$

This composition must have the form

$$
\psi_{j} \psi_{i}^{-1}\left(x,\left[\begin{array}{c}
a^{1} \\
\vdots \\
a^{n}
\end{array}\right]\right)=\left(x, \gamma_{i j}(x),\left[\begin{array}{c}
a^{1} \\
\vdots \\
a^{n}
\end{array}\right]\right),
$$

where $\gamma_{i j}(x) \in G l(n), \forall x \in W_{i} \cap W_{j}$.

Lemma 5.1. The map $\gamma_{i j}: W_{i} \cap W_{j} \rightarrow G l(n)$ is smooth.

Proof: Let $e_{k}$ denote the column vector with 0's in all places except the $k^{\text {th }}$, where the entry 1 . Then $e_{k}(x)=\psi_{j} \psi_{i}^{-1}\left(x, e_{k}\right)$ defines a smooth map

$$
e_{k}: W_{i} \cap W_{j} \rightarrow\left(W_{i} \cap W_{j}\right) \times \mathbb{R}^{n}
$$

$1 \leq k \leq n$. But $e_{k}(x)=\psi_{j} \psi_{i}^{-1}\left(x, \gamma_{i j}(x), e_{k}\right)$ and the second entry is just the $k^{t h}$ column of $\gamma_{i j}(x)$. Since $k$ is arbitrary, $1 \leq k \leq n$, we see that the $n^{2}$ entries of $\gamma_{i j}(x)$ are the smooth functions of $x$.

Definition 5.2. The smooth maps have the 'cocycle' property

$$
\text { (i) } \gamma_{k j}(x) \cdot \gamma_{j i}(x)=\gamma_{k i}(x)
$$


Global Theory of Smooth Functions of Manifolds

$\forall x \in W_{i} \cap W_{j} \cap W_{k} \forall i, j, k \in J$. As usual, this property implies the following also, for all appropriate choice of $x$ and indices $i, j \in J$.

$$
\begin{aligned}
\text { (ii) } \gamma_{i i}(x) & =I_{n} \\
\text { (iii) } \gamma_{i j}(x) & =\left(\gamma_{j i}(x)\right)^{-1}
\end{aligned}
$$

Definition 5.3. A $G l(n)$ - cocycle property [4] on $M$ is a family $\gamma=\left\{W_{j}, \gamma_{i j}\right\}_{i, j \in J}$ such that $\left\{W_{j}\right\}_{j \in J}$ is an open cover of $M$ and $\gamma_{j i}: W_{i} \cap W_{j} \rightarrow G l(n)$ is a smooth map, $\forall i, j \in J$, all subject to the cocycle condition property $(i)$ of definition 4.02. if the cocycle $\gamma$ arises as above from an $n$-plane bundle $E$, it is said to be a structure cocycle of $E$.

Definition 5.4. Two $G l(n)$-cocycles

$$
\gamma=\left\{W_{j}, \gamma_{i j}\right\}_{i, j \in J} \text { and } \theta=\left\{V_{a}, \theta_{a b}\right\}_{a, b \in A}
$$

On the same manifold $M$ equivalent if they are contained in a common $G l(n)$-cocycle on $M$. The equivalence class of $\gamma$ will be denoted by $[\gamma]$.

Corollary 5.5. Equivalence $G l(n)$-cocycles is an equivalence relation.

Proof: If $\gamma \sim \theta$ and $\theta \sim \delta$, let $\psi$ be a cocycle containing both $\gamma$ and $\theta, \varphi$ be cocycle containing both $\theta$ and $\delta$. Then $\psi$ and $\varphi$ both contain $\theta$. We know the theorem "If two $G l(n)$-cocycles on same manifold contain a common $G l(n)$-cocycle, then they are contained in a common $G l(n)$-cocycle" from this theorem guarantees that they are contained in a common cocycle $\rho$. Then $\gamma \subseteq \rho$ and $\delta \subseteq \rho$, so $\gamma \sim \delta$.

Theorem 5.6. If $\gamma$ is a $G l(n)$-cocycle on $M$, the isomorphism class $E[\gamma] \in \operatorname{Vect}_{n}(M)$ depends only one equivalence class $[\gamma] \in H^{1}(M ; G l(n))$. This defines a canonical bijective correspondence

$$
\operatorname{Vect}_{n}(M) \mapsto H^{1}(M ; G l(n))
$$

where $\operatorname{Vect}_{n}(M)$ denote the set of isomorphism classes $[E]$ of $n$-plane bundles $E$ on $M$ and $H^{1}(M ; G l(n))$ denote the equivalence classes of $G l(n)$-cocycles.

Proof: In this case we have to identify $\operatorname{Vect}_{n}(M)$ to $H^{1}(M ; G l(n))$. The tangent bundle

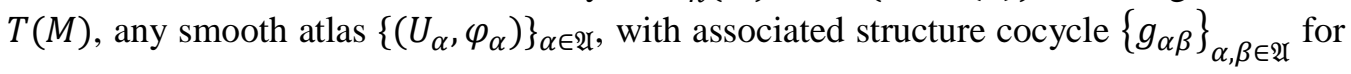

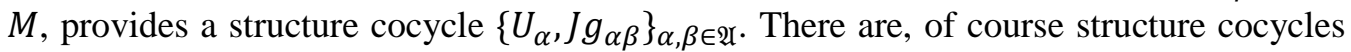
for $T(M)$ that are not obtained in this way, but these special cocycles tie together the bundle structure of $T(M)$ and the smooth structure of $M$.

Definition 5.7. A structure cocycle $\left\{U_{\alpha}, J g_{\alpha \beta}\right\}_{\alpha, \beta \in \mathfrak{A}}$ for $T(M)$, associated to smooth atlas on $M$, will be called a Jacobian cocycle. If $T(M)$ admits a Jacobian cocycle such that $J g_{\alpha \beta}=I_{n}, \forall \alpha, \beta \in \mathfrak{U}$, then $M$ is said to be integrably parallelizable.

Definition 5.8. Let $G \subseteq G l(n)$ be a subgroup and let $\pi: E \rightarrow M$ be an $n$-plane bundle. We say that the structure group of $E$ can be reduced to $G$ if there is a $G l(n)$-cocycle $\left\{W_{j}, \gamma_{j i}\right\}_{i, j \in J}$ representing the isomorphism class of $E$ such that $\left(\gamma_{j i}\right) \subseteq G, \forall j, i \in J$. Such a cocycle will be called a $G$-cocycle for $E$. 
M.R.Hossain, Md. Raknuzzaman, Md. Mijanoor Rahman and Md. Jamal Hossain

\section{Global constructions of smooth functions}

Proposition 6.1. Let $M$ be an $n$-manifold, let $U \subseteq M$ be an open subset and $K \subset U$ a compact subset. Then there is a smooth function $f: M \rightarrow \mathbb{R}$ such that $f \mid K \equiv 1$ and $\operatorname{supp}(f) \subset U$.

Proof: For each $p \in K$, choose a coordinate neighborhood $\left(U_{p}, z_{p}\right)$ about $p, U_{p} \subseteq U$, and an open, $n$-dimensional interval $A_{p}$ with $\bar{A}_{p} \subset U_{p}$, centered at $p$. Since $K$ is compact, finitely many of the $A_{p}$ cover $K$.

Lemma 6.2. Let $M$ be a smooth manifold and let $x \in M$. Then the natural map

that carries $f \rightarrow[f]_{x}$ is surjective.

$$
C^{\infty}(M) \rightarrow \mathfrak{B}_{x}
$$

Proof: Given $[g]_{x} \in \mathfrak{B}_{x}$, find $\varphi \in C^{\infty}(M)$ with $\operatorname{supp}(\varphi) \subset \operatorname{dom}(g)$ and $\varphi \equiv 1$ on some compact neighborhood of $x$ in $\operatorname{dom}(g)$. Then $\varphi g$ extends by 0 to the smooth function $f$ on $M$ and $[f]_{x}=[g]_{x}$.

Proposition 6.3. If $\mathcal{U}=\left\{U_{\alpha}\right\}_{\alpha \in \mathfrak{A}}$ is an open cover of $M$, there is a $C^{\infty}$ partition of unity $\left\{\lambda_{\alpha}\right\}_{\alpha \in \mathfrak{A}}$ subordinate to $\mathcal{U}$.

Proof: First remark that $\mathcal{V}=\left\{V_{\beta}\right\}_{\beta \in \mathfrak{B}}$ is a refinement of unity subordinate to $\mathcal{V}$ induces a smooth partition of unity subordinate of $\mathcal{U}$. Indeed, let $i: \mathfrak{B} \rightarrow \mathfrak{A}$ be a map such that $V_{\beta} \subseteq U_{i(\beta)}, \forall \beta \in \mathfrak{B}$. If $\left\{\mu_{\beta}\right\}_{\beta \in \mathfrak{B}}$ is a partition of unity subordinate to $\mathcal{V}$, define $\lambda_{\alpha}=$ $\sum_{\beta \in i^{-1}(\alpha)} \mu_{\beta}, \forall \alpha \in \mathfrak{A}$. If $i^{-1}(\alpha)=\emptyset$, we understand that $\lambda_{\alpha} \equiv 0$. It is clear that $\left\{\lambda_{\alpha}\right\}_{\alpha \in \mathfrak{A}}$ is a partition of unity subordinate to $\mathcal{U}$.

By passing to a suitable locally finite refinement of $\mathcal{U}$ and applying the above paragraph, we lose no generality in assuming that

(i) $\mathcal{U}$ is locally finite.

(ii) each $U_{\alpha} \in \mathcal{U}$ is the domain of a $C^{\infty}$ coordinate chart $\left(U_{\alpha}, \varphi_{\alpha}\right)$;

(iii) there are open $n$-dimensional intervals $A_{\alpha}$ with $\bar{A}_{\alpha} \subset \varphi_{\alpha}\left(U_{\alpha}\right)$ compact and such that $\left\{\varphi_{\alpha}^{-1}\left(A_{\alpha}\right)\right\}_{\alpha \in \mathfrak{A}}$ covers $M$.

By Proposition 5.01, define $f_{\alpha} \in C^{\infty}(M)$ in such a way that $f_{\alpha} \mid \varphi_{\alpha}^{-1}\left(\bar{A}_{\alpha}\right) \equiv 1$ and $\operatorname{supp}\left(f_{\alpha}\right) \subset U_{\alpha}$. Since the cover $\left\{U_{\alpha}\right\}_{\alpha \in \mathscr{N}}$ is locally finite, we can define

$$
f=\sum_{\alpha \in \mathfrak{A}} f_{\alpha} \in C^{\infty}(M),
$$

remarking that $f>0$ on $M$. We obtain the smooth partition of unity by setting $\lambda_{\alpha}=$ $f_{\alpha} / f, \forall \alpha \in \mathfrak{A} . \square$

Proposition 6.4. If $M$ is a smooth manifold and $X \subseteq M$, then

$$
f: X \rightarrow \mathbb{R}^{k}
$$

is smooth if and only if $\forall x \in X, \exists$ an open neighborhood $U_{x} \subseteq M$ of $x$, and a smooth map $f_{x}: U_{x} \rightarrow \mathbb{R}^{k}$ such that $f_{x}\left|\left(U_{x} \cap X\right)=f\right|\left(U_{x} \cap X\right)$.

Proof: This property clearly follows from our definition of smoothness. We must recover our definition from this property. Let $U=\cup_{x \in X} U_{x}$. Then there is a must partition of unity $\left\{\lambda_{x}\right\}_{x \in X}$ on $U$, subordinate to the open cover $\left\{U_{x}\right\}_{x \in X}$ of the manifold $U$. Since each $f_{x}$ in $\mathbb{R}^{k}$-valued, $\lambda_{x} f_{x}$ makes sense and can be interpreted as a smooth map of $U$ into $\mathbb{R}^{k}$. Then define

$$
\tilde{f}=\sum_{x \in X} \lambda_{x} f_{x},
$$


Global Theory of Smooth Functions of Manifolds

a smooth map of $U$ into $\mathbb{R}^{k}$. Evidently

$$
\tilde{f}(y)=\left(\sum_{x \in X} \lambda_{x}(y)\right) f(y)=1 . f(y)=f(y),
$$

$\forall y \in X$, so $\tilde{f}$ is the required smooth extension of $f$ to the neighborhood $U$ of $X$.

Definition 6.5. A function $f: X \rightarrow Y$ from the subset $X \subseteq M$ of smooth manifold [7] $M$ into the subset $Y \subseteq N$ of a smooth manifold $N$ is said to be smooth if, for each $x \in X$, there is a open neighborhood $U_{x} \subseteq M$ of $x$ and smooth map $f_{x}: U_{x} \rightarrow N$ such that $f_{x}\left|\left(U_{x} \cap X\right)=f\right|\left(U_{x} \cap X\right)$. Such a map is diffeomorphism of $X$ onto $Y$ if it is bijective and both $f$ and $f^{-1}$ are smooth.

Theorem 6.6. Let $M$ and $N$ be $C^{\infty}$ manifolds of the same dimension $n$. If $U \subseteq M$ is open, if $X \subseteq N$ and if $\varphi: U \rightarrow X$ is a diffeomorphism, then $X$ is open in $N$.

Proof: Let $x_{0} \in U$ and $\varphi\left(x_{0}\right) \in X$. Since $\varphi^{-1}: X \rightarrow U$ is smooth, there is an open neighborhood $V$ of $\varphi\left(x_{0}\right)$ in $N$ and a smooth extension $\psi: V \rightarrow \mathbb{R}^{n}$ of $\varphi^{-1} \mid(V \cap X)$. Since $\varphi: U \rightarrow X$ is continuous, $\widetilde{V}=\varphi^{-1} \mid(V \cap X)$ is an open neighborhood of $x_{0}$ in $U$ and

$$
\psi \circ \varphi\left|\tilde{V}=\varphi^{-1} \circ \varphi\right| \tilde{V}=i d_{\widetilde{V}} .
$$

Since $\varphi: U \rightarrow N$ is smooth in the usual sense, the chain rules gives

$$
d \psi_{\varphi\left(x_{0}\right)} \circ d \varphi_{x_{0}}=i d_{T_{x_{0}}(M)} \text {, }
$$

so $d \varphi_{x_{0}}: T_{x_{0}}(M) \rightarrow T_{\varphi\left(x_{0}\right)}(N)$ is a linear isomorphism. By the inverse function theorem, there is a open neighborhood $W \subseteq \tilde{V} \subseteq U$ of $x_{0}$ which is carried by $\varphi$ diffeomorphically onto an open subset $\varphi(W) \subseteq N$. But $\varphi\left(x_{0}\right)$ is an arbitrary point of $X$ and $\varphi\left(x_{0}\right) \in$ $\varphi(W) \subseteq X$, so $X$ is an open subset of $N$.

\section{Manifolds with boundary}

Since Euclidean half space $\mathbb{H}^{n}$ is a subset of smooth manifold $\mathbb{R}^{n}$, definition 5.05 allows us to talk about a smooth maps and diffeomorphism [8] between open subsets of $\mathbb{H}^{n}$. Thus, if $M$ is a topological manifold with boundary, it makes sense to talk about two $\mathbb{H}^{n}$ charts on $M$ being $C^{\infty}$-related, so we can define a differentiable structure on $M$ to be a maximal $\mathbb{H}^{n}$-atlas $\mathcal{A}$ of $C^{\infty}$-related charts. As in the topological case, we define

$$
\begin{gathered}
\partial M=\left\{x \in M \mid \exists\left(U_{\alpha}, \varphi_{\alpha}\right) \in \mathcal{A}, x \in U_{\alpha}, \varphi_{\alpha}(x) \in \partial \mathbb{H}^{n}\right\} \\
\operatorname{int}(M)=\left\{x \in M \mid \exists\left(U_{\alpha}, \varphi_{\alpha}\right) \in \mathcal{A}, x \in U_{\alpha}, \varphi_{\alpha}(x) \in \operatorname{int}\left(\mathbb{H}^{n}\right)\right\}
\end{gathered}
$$

The pair $(M, \mathcal{A})$ is a (smooth) $n$-manifold with boundary $\partial M$. Of course, all smooth $n$ manifolds without boundary are special cases, as is $\mathbb{H}^{n}$ itself. The notation of smooth maps between manifolds with boundary is defined exactly as in the boundaryless case.

Definition 7.1. If $f: M \rightarrow N$ and $g: N \rightarrow P$ are smooth maps between manifolds with boundary, then $g \circ f$ is smooth and for each $x \in M$.

$$
d(g \circ f)_{x}=d g_{f(x)} \circ d f_{x}
$$

Lemma 7.2. Let $x \in \partial \mathbb{H}^{n}$ and let $\rho: \mathfrak{B}_{x}\left(\mathbb{R}^{n}\right) \rightarrow \mathfrak{B}_{x}\left(\mathbb{H}^{n}\right)$ be defined by $\rho[f]_{x}=$ $\left[f \mid\left(\mathbb{H}^{n} \cap \operatorname{dom}(f)\right)\right]_{x}$. Then $\rho$ is a surjection.

Proof: Let $U \subseteq \mathbb{H}^{n}$ be an open neighborhood of $x$. If $g: U \rightarrow \mathbb{R}$ is smooth, there is a neighborhood $V$ of $x$ in $\mathbb{R}^{n}$ and a smooth extension $\tilde{g}: V \rightarrow \mathbb{R}$ of $g \mid\left(V \cap U \cap \mathbb{H}^{n}\right)$. Then $[\tilde{g}]_{x} \in \mathfrak{B}_{x}\left(\mathbb{R}^{n}\right)$ and $\rho[\tilde{g}]_{x}=[g]_{x}$. 
M.R.Hossain, Md. Raknuzzaman, Md. Mijanoor Rahman and Md. Jamal Hossain

Lemma 7.3. For $x \in \partial \mathbb{H}^{n}$, define $\rho^{*}: T_{x}\left(\mathbb{H}^{n}\right) \rightarrow T_{x}\left(\mathbb{R}^{n}\right)$ then by setting $\rho^{*}(D)[f]_{x}=$ $D\left(\rho[f]_{x}\right)$. Then we have have to show that $\rho^{*}$ is bijective.

Proof: We prove that $\rho^{*}$ is one to one. If $\rho^{*}\left(D_{1}\right)=\rho^{*}\left(D_{2}\right)$, then $D_{1}\left(\rho[f]_{x}\right)=$ $D_{2}\left(\rho[f]_{x}\right), \forall[f]_{x} \in \mathfrak{B}_{x}\left(\mathbb{R}^{n}\right)$. Since $\rho$ is surjective, it follows that $D_{1}[g]_{x}=$ $D_{2}[g]_{x}, \forall[f]_{x} \in \mathfrak{B}_{x}\left(\mathbb{H}^{n}\right)$, so $D_{1}=D_{2}$.

We have to prove that $\rho^{*}$ is onto. Let $v \in T_{x}\left(\mathbb{R}^{n}\right)=\mathbb{R}^{n}$. As an infinitesimal curve, this vector is represented by $s(t)=x+t v$. As an operator on germs, $v=D_{\langle s\rangle_{x}}$. Either $v$ points into $\mathbb{H}^{n}$ (we intend this to include the case that $v$ is tangent to $\partial \mathbb{H}^{n}$ ) or $v$ points out of $\mathbb{H}^{n}$, in this case $-v$ points into $\mathbb{H}^{n}$.

If $v$ points into $\mathbb{H}^{n}$, then $s(t) \in \mathbb{H}^{n}, t \geq 0$. Define $D: \mathfrak{B}_{x} \rightarrow \mathbb{R}$ by

$$
D[g]_{x}=\lim _{t \rightarrow 0^{+}} \frac{g(s(t))-g(x)}{t}
$$

It is elementary that $D \in T_{x}\left(\mathbb{H}^{n}\right)$ and that $\rho^{*}(D)=D_{\langle s\rangle_{x}}=v$.

If $v$ points out of $\mathbb{H}^{n}$, then $s(t) \in \mathbb{H}^{n}, \forall t \leq 0$. Define $D: \mathfrak{B}_{x}\left(\mathbb{H}^{n}\right) \rightarrow \mathbb{R}$ by

$$
D[g]_{x}=\lim _{t \rightarrow 0^{-}} \frac{g(s(t))-g(x)}{t}
$$

Again, $D \in T_{x}\left(\mathbb{H}^{n}\right)$ and $\rho^{*}(D)=v$.

\section{Submanifolds}

Let $M$ be an $m$-manifold, possibly with the boundary. A subset $X \subset M$ is a properly imbedded submanifold [1] of dimension $n$ if and only if, $\forall p \in X$, there is an $\mathbb{H}^{m}$ coordinate chart $(U, \varphi)$ about $p$ in $M$ in which $\varphi(U \cap X)=\varphi(U) \cap \mathbb{H}^{n}$, where $\mathbb{H}^{n} \subset$ $\mathbb{H}^{m}$ is the (image of the) standard inclusion.

Remark that, in the above definition $(U \cap X, \varphi \mid(U \cap X))$ can be viewed as an $\mathbb{H}^{n}$ coordinate chart on $X$ and that the collection of all such charts makes $X$ a smooth $n$ manifold with boundary $\partial X=X \cap \partial M$. Thus if $\partial M=\emptyset$, then $\partial X=\emptyset$ also.

Theorem 8.1. Let $f: M \rightarrow N$ be a smooth map between the manifolds of respective dimensions $m$ and $n$. Assume that $\partial N=\emptyset$ and that $m>n$. If $y \in N$ is a regular value simultaneously for $f$ for $\partial f=f \mid \partial M$, then $f^{-1}(y)$ is a properly imbedded submanifold of dimension $m-n$.

Proof: Let $p \in f^{-1}(y)$ and the find a suitable coordinate chart about $p$ in $M$. There are two cases.

Case 1. Suppose $p \in \operatorname{int}(M)$. Choose a coordinate neighborhood $(U, x)$ about $p$ such that $U \subseteq \operatorname{int}(M)$. Then $y$ is a regular value of $f \mid U$ so it implies that $f^{-1}(y) \cap U$ is a smooth submanifold of $U$ of a dimension $m-n$.

Case 2. Suppose $p \in \partial M$ and let $\left(U, x^{1}, x^{2}, \ldots \ldots, x^{m}\right)$ be an $\mathbb{H}^{m}$ charts about $p$ in $M$. Assume that $f(u) \subset W$, where $\left(W, y^{1}, y^{2}, \ldots \ldots, y^{n}\right)$ is a coordinate chart about $y$ in which $y=0$. Let $\partial f \mid(U \cap \partial M)$ be denoted by $\varphi\left(x^{2}, \ldots \ldots, x^{m}\right)$ with component functions $\varphi^{1}, \ldots \ldots, \varphi^{n}$ relative to the coordinate of $W$. Since $p$ is a regular point for $\varphi, U$ can be chosen so small that the matrix

$$
\left[\begin{array}{ccc}
\frac{\partial \varphi^{1}}{\partial x^{2}} \ldots \cdots & \frac{\partial \varphi^{1}}{\partial x^{m}} \\
\vdots & \vdots \\
\frac{\partial \varphi^{n}}{\partial x^{2}} \ldots & \cdots & \frac{\partial \varphi^{n}}{\partial x^{m}}
\end{array}\right]
$$


Global Theory of Smooth Functions of Manifolds

has constant rank $n$ on $U \cap \partial M$. By permutation of coordinates $x^{2}, \ldots \ldots, x^{m}$, it can be assumed that the last $n \times n$ block

$$
\left[\begin{array}{cc}
\frac{\partial \varphi^{1}}{\partial x^{m-n+1}} \ldots \ldots & \frac{\partial \varphi^{1}}{\partial x^{m}} \\
\vdots & \\
\frac{\partial \varphi^{n}}{\partial x^{m-n+1}} \ldots \ldots & \frac{\partial \varphi^{n}}{\partial x^{m}}
\end{array}\right]
$$

is non singular on $U \cap \partial M$. Choosing $U$ even smaller, if necessary the corresponding $n \times n$ block in the matrix

$$
\left[\begin{array}{cccc}
\frac{\partial f^{1}}{\partial x^{1}} & \frac{\partial f^{1}}{\partial x^{2}} \ldots \ldots & \frac{\partial f^{1}}{\partial x^{m}} \\
\vdots & \vdots & \vdots \\
\frac{\partial f^{n}}{\partial x^{1}} & \frac{\partial f^{n}}{\partial x^{2}} \ldots \ldots & \frac{\partial f^{n}}{\partial x^{m}}
\end{array}\right]
$$

is also nonsingular. We then resort to the trick of recoordinatizing $U$ near $p$ by setting $z^{i}=x^{i}, 1 \leq i \leq m-n$, and $z^{m-n+j}=f^{j}, 1 \leq j \leq n$. The inverse function theorem shows, by the above remarks, that this will define an $\mathbb{H}^{n}$ chart on a small enough neighborhood (again called $U$ ) of $p$. But, relative to these coordinates,

$$
f\left(z^{1}, z^{2}, \ldots \ldots, z^{m}\right)=\left(z^{m-n+1}, \ldots \ldots, z^{m}\right)
$$

Then $f^{-1}(y) \cap U$ is the set of points with coordinates $\left(z^{1}, \ldots \ldots, z^{m}, 0, \ldots \ldots, 0\right)$. That is

$$
f^{-1}(y) \cap U=\mathbb{H}^{m-n} \cap U .
$$

Example 8.2. Let $f: \mathbb{H}^{n+1} \rightarrow \mathbb{R}$ be given by

$$
f\left(x^{1}, x^{2}, \ldots \ldots, x^{n+1}\right)=\sum_{i=1}^{n+1}\left(x^{i}\right)^{2}
$$

Then $1 \in \mathbb{R}$ is a singular value both for $f$ and for $\partial f$. The hemisphere $f^{-1}(1)$ is the intersection $S^{n} \cap \mathbb{H}^{n+1}$ and is an $n$ - manifold with boundary [4] $f^{-1}(1) \cap \partial \mathbb{H}^{n+1}=$ $S^{n-1}$.

Lemma 8.4. If $\partial M=\emptyset$ and $f: N \rightarrow M$ is smooth, then the set of points in $M$ that are simultaneous regular values for $f$ and $\partial f$ in dense in $M$.

Proof: Clearly, if $p \in \partial N$ is a regular point for $\partial f$, it is also the regular point for $f$. Thus $y \in M$ is a regular value both of $f$ and $\partial f$ precisely when it is a regular value both of $f \mid \operatorname{int}(N)$ and of $\partial f$. Use countable coordinate coverings $\left\{U_{i}\right\}_{i \in I}$ of $\operatorname{int}(N),\left\{V_{j}\right\}_{j \in J}$ of $\partial N$, and $\left\{W_{k}\right\}_{k \in K}$ of $M$. For each $k \in K$, consider the countable family of smooth maps

$$
\begin{gathered}
f_{i k}: U_{i} \cap f^{-1}\left(W_{k}\right) \rightarrow W_{k} \\
\partial f_{j k}: U_{j} \cap \partial f^{-1}\left(W_{k}\right) \rightarrow W_{k}
\end{gathered}
$$

Obtained by restrictions. So we have $y \in W_{k}$ is a common regular value of all these maps. Doing this for each $k \in K$, we complete proof.

\section{REFERENCES}

1. F.Brickell and Clark, Differential Manifolds, Van Nostrand Reinhold Company, London, R.S.1970

2. P.J.Hilton and S.Wylie, Homology Theory, Cambridge University Press, 1962.

3. S.Kobayashi and K.Nomizu, Foundations of Differential Geometry, Vol. I, Interscience, 1969. 
M.R.Hossain, Md. Raknuzzaman, Md. Mijanoor Rahman and Md. Jamal Hossain

4. S.Kobayashi and K.Nomizu, Foundations of Differential Geometry, Vol. II, Interscience, 1969.

5. R.O.Wells, Differential Analysis on Complex Manifolds, Springer Verlag, 1979.

6. E.H.Spanier, Algebraic Topology, McGraw-Hill, 1966.

7. N.Steenrod, The Topology of Fibre Bundles, Princeton University Press, 1970.

8. C.Chevally, Theory of Lie Groups, Princeton University Press, 1946.

9. J.W.Milnor and J.D.Stasheff, Characteristic Classes, Princeton University Press, 1974.

10. N.Steenrod, The Topology of Fibre Bundles, Princeton University Press, 1970.

11. W.S.Massey, Singular Homology Theory, Springer Verlag, 1980.

12. F.W.Warner, Foundations Of Differentiable Manifolds and Lie Groups, Springer, 1983.

13. W.Poor, Differential Geometric Structures, McGraw-Hill, New York (1981)

14. W.Ballmann, G.Thorbergsson and W.Ziller, Closed geodesics on positively curved manifolds, Ann. Math., 116(2) (1982) 213-247.

15. R.Bott and L.W.Tu, Differential Forms in Algebraic Topology, Springer-Verlag, 1982.

16. M.P.do Carmo, Differential Forms and Applications, Springer Verlag, 1994.

17. S.S.Chern, ed., Global Geometry and Analysis, 2nd edition, MAA Studies 27, Washington: Mathematical Association of America, 1989.

18. P.Michor, A convenient setting for differential geometry and global analysis, Cahiers de Top. et, Geom. Diff., 25 (1984) 63-109.

19. A.Frölicher, Cartesian closed categories and analysis of smooth maps, Seminarberichte Fernuniversität Hagen, 160 (1982) 5-16. 